\title{
T regulatory cells in childhood arthritis - novel insights
}

\section{Anne M. Pesenacker and Lucy R. Wedderburn*}

In recent years, there have been many new developments in the field of regulatory T cells (Treg), challenging the consensus on their behaviour, classification and role(s) in disease. The role Treg might play in autoimmune disease appears to be more complex than previously thought. Here, we discuss the current knowledge of regulatory $T$ cells through animal and human research and illustrate the recent developments in childhood autoimmune arthritis (juvenile idiopathic arthritis (JIA)). Furthermore, this review summarises our understanding of the fields and assesses current and future implications for Treg in the treatment of JIA.

\section{Introduction}

Evidence indicates that in health regulatory T cells (Treg) play an important role in the maintenance of immunological tolerance and prevention of autoimmune diseases by the regulation of immune responses. Juvenile idiopathic arthritis (JIA), the most common inflammatory rheumatic disease in children, comprises of a group of childhood conditions characterised by the onset of arthritis before the age of 16. JIA has a predominance among girls for most forms of JIA. There are seven main subtypes of JIA according to the International League of Associations for Rheumatology (ILAR) classification (Ref. 1). Each subtype is clinically distinct with respect to the presenting symptoms, complications and prognosis. Oligoarticular arthritis (O-JIA), defined as arthritis that affects four joints or fewer in the first 6 months, is the most common form of JIA, whereas polyarticular JIA (P-JIA), affecting five or more joints, is the second most common subtype. Conflicting reports regarding the frequency and functioning of Treg found in the blood of children with JIA have been published. However, a consistent finding by several studies is a clear enrichment of Treg within the synovial fluid (SF) infiltrate in the joints of children with JIA compared with the level observed in peripheral blood (PB) (Refs 2, 3).

The presence of high numbers of Treg in the joint appears to present a paradox, since arthritis still persists despite the presence of this regulatory population. One theory to explain this paradox is that the proinflammatory milieu of the joint may inhibit the ability of regulatory $\mathrm{T}$ cells to abrogate inflammation efficiently. In addition, there are studies demonstrating that effector T cells in the joint are relatively resistant to suppression (Refs 4,5). In this review, we will discuss recent advances in the field of Treg and how this knowledge improves our understanding of JIA pathogenesis and ultimately may help to optimise clinical treatments for these diseases.

\section{Emergence of regulatory T cells}

A central function of the immune system is to maintain tolerance to self-tissues and molecules and thereby prevent autoimmune disease. It was

Rheumatology Unit, UCL Institute of Child Health, University College London, London, UK *Corresponding author: Lucy R Wedderburn, Rheumatology Unit, UCL Institute of Child Health, University College London, 30 Guilford Street, London WC1N 1EH, UK. E-mail: 1.wedderburn@ ucl.ac.uk 
over 40 years ago that the concept of suppression of immune responses and its contribution to the maintenance of tolerance was first proposed. However, with the identification of markers that are expressed on regulatory $\mathrm{T}$ cells, the investigation of these cells has gained huge pace. It was first discovered that inoculation of athymic (nude/nude) mice with CD4+ $\mathrm{T}$ cells, which were depleted of CD25+ cells, led to severe autoimmunity, which could be inhibited by the addition of CD4 + CD25+ cells (Ref. 6). Similarly, in a diabetes rat model, disease was prevented by using CD4 + CD25+ cells and accelerated by CD4 + CD25- cells (Ref. 7). Furthermore, CD4 + CD25+ cells could also inhibit polyclonal CD4 + CD25- T cells in vitro (Ref. 8). However, even early studies demonstrated that not all CD4+ CD25+ T cells have suppressive capacity (Ref. 9).

Later, several groups described CD4 + CD25+ regulatory cells in human blood, which were functional in vitro (Refs 10,11,12). It was also determined that the CD4 + CD25+ cells were anergic when stimulated alone in vitro, produced little inflammatory cytokines such as interleukin (IL)-2 and interferon gamma (IFN- $\gamma$ ), and in vitro suppression could be overcome by addition of exogenous IL-2 (Refs 10, 11, 12, 13). However, these observations indicated that human CD4 + CD25+ T cells are a heterogeneous population, which may include both suppressive and non-suppressive cells. This might, in part, be because of the upregulation of CD25 expression, the $\alpha$-chain of the IL-2 receptor, upon division or activation of human $\mathrm{T}$ cells. The demonstration that very high expression levels of CD25 correlated with good suppression (Refs 11, 14) led to the proposal that the CD25igh population contains the most suppressive cells.

Together with the identification of $\mathrm{CD} 25^{\text {high }}$ cells being suppressive, severe autoimmunity in the murine model scurfy was linked to mutations in the Foxp3 gene (Ref. 15). In humans, the FOXP3 gene was shown to be affected in the severe autoimmune condition IPEX (immunodysregulation polyendocrinopathy enteropathy X-linked syndrome) (Ref. 16). Further research demonstrated that FoxP3 was crucial for the development of Treg and their function (Refs 17, 18, 19, 20). Together, these studies established FoxP3 as a master transcription factor for Treg. The development of specific monoclonal antibodies to identify FoxP3 $+\mathrm{T}$ cells by standard assays such as flow cytometry, combined with the definition of other typical phenotypic features for these cells (such as the low level of IL-7R $\alpha$ (CD127)), has facilitated the growth of research in this area. Some controversies exist about the available reagents to detect FoxP3 protein, but with appropriate controls reliable detection of FoxP3 protein is now routine (Ref. 21). More recently, evidence that the stable expression of FoxP3 is dependent upon demethylation at specific $5^{\prime}$ regions of the FOXP3 locus, the Treg-specific demethylated region (TSDR), has led to a further method by which to identify bona fide regulatory $\mathrm{T}$ cells (Refs 22, 23, 24). These studies have also shown that cells that express FoxP3 transiently are not demethylated at this region.

\section{Not all Treg are equal}

Regulatory $\mathrm{T}$ cells can develop in the thymus (natural Treg) or be induced in the periphery (induced or adaptive Treg). The IKAROS family member Helios has been proposed as a possible marker to distinguish between natural and induced Treg, as some reports suggest that it is uniquely expressed by natural Treg (Ref. 25). However, upon further investigation, Helios expression could also be detected in induced Treg under certain conditions (Refs 26, 27). In mice non-coding DNA sequences (CNS) at the FOXP3 locus appear to control differentially thymic and peripheral FoxP3 expression, with CNS3 necessary for thymic, CNS1 for peripheral FoxP3 expression and CNS2 for FoxP3 expression stability (Ref. 28), it remains to be seen if these conserved CNS regions also control FoxP3 expression in human Treg. Therefore, to date there is no generally accepted way to distinguish between natural and induced Treg, and functionally, both natural and induced Treg can suppress aberrant responses as shown in different transgenic and adoptive transfer mouse models (Refs 29, 30, 31, 32).

Nowadays, it is becoming clearer that the CD4+ FoxP3+ Treg population, typically $5-10 \%$ of human peripheral blood CD4 T cells, is in fact, a heterogeneous population, which differs in terms of phenotype and possibly function. Various strategies to divide Treg into different subsets have been used. One proposed subdivision was based on the expression of the major histocompatibility complex (MHC) class II molecule DR (HLA-DR), which is also expressed on activated $\mathrm{T}$ cells (Refs 33, 34). Typically, 
$20-30 \%$ of Treg express HLA-DR (Ref. 35) and functional studies revealed that HLA-DR+ Treg exert early contact-dependent suppression. HLA-DR - Treg, on the other hand, skew cytokine production to a Th2-like phenotype and may also suppress through contactdependent mechanisms (Ref. 36).

Treg can also be subdivided by expression of markers typical of naïve or memory $\mathrm{T}$ cells. Memory Treg, with the phenotype CD45RA CD45RO+ and expression of CCR6 (Refs 37, 38, 39), express high levels of the functional surface molecules CTLA4 and CD39, the effector memory marker CD44, but low levels of CD62L. In comparison with naïve Treg, it has been suggested that memory Treg have a higher turnover, suggesting that a greater proportion is in cell cycle, both in vitro (Ref. 39) and in vivo (Refs 37, 40). CTLA4 and CD39 are both important in Treg function (Refs 41, 42, 43). The exact role of CTLA4 in Treg function is not yet fully understood, but theories include that CTLA4 competes with CD28 for co-stimulatory receptors which in turn provides a negative signal for $\mathrm{T}$ cell activation, or it may act in a $\mathrm{T}$ cell-extrinsic manner such as by stripping costimulatory molecules from antigen-presenting cells (Ref. 44).

CD39 is a potent cell-surface ATPase, which breaks down proinflammatory ATP to AMP. CD73 in turn breaks down AMP to adenosine. In mice, both these enzymes are expressed on Treg (Ref. 45). In humans, however, there is conflicting evidence, whereas some researchers report CD73 and CD39 expression on Treg (Ref. 46), while we have observed that CD73 is not co-expressed with CD39 on Treg (Ref. 47). Owing to the pro-inflammatory actions of ATP in the extracellular milieu, ATP breakdown is thought to contribute to Treg function.

CCR6 is an important chemokine receptor, and a characteristic hallmark of Th17 cells, but it might also be important in directing Treg to the site of inflammation (Refs 48, 49). Treg can also express a variety of other chemokine receptors, associated with different Th lineages (Refs 50, 51, $52,53,54)$. Specific chemokine receptor expression may enable specific co-localisation of certain Treg with specific T helper lineages (Ref. 52, 54).

Treg can express many activation markers, which are shared with conventional CD4+ T cells (Tconv), such as CD44, CD69 and GITR. Recently, a Treg-specific activation marker has been demonstrated, the glycoprotein A repetitions predominant (GARP) protein (Refs 55, 56). GARP is expressed on Treg recently activated through the TCR, but not on Tconv, or TGF- $\beta$ induced Treg. Interestingly, GARP serves as a receptor for latent TGF- $\beta$, a cytokine known to promote Treg development and stability (Ref. 57). GARP overexpression in helper $\mathrm{T}$ cells induces an efficient reprogramming of effector $\mathrm{T}$ cells into Treg although these reprogrammed cells are not fully demethylated at the TSDR (Ref. 58).

\section{Treg may display effector cell functions}

Treg can express a variety of memory markers as well as chemokine receptors that are associated with a Tconv phenotype. It has been proposed that expression of a specific transcription factor commonly associated with Tconv may 'assist' specific Treg to target its suppressive capacity towards specific Tconv and may even be absolutely required for such suppression. For example, Treg suppression of Th1 cells is dependent on Tbet expression (Ref. 52), IRF4 expression is crucial for Th2-targeting Treg (Ref. 59), whereas STAT3 is essential for Treg suppression of Th17 (Refs 60, 61). Furthermore, BCL6 positive Treg are important in controlling follicular helper $\mathrm{T}$ and $\mathrm{B}$ cells in the germinal centre (Refs 62, 63).

Interestingly, there is now increasing evidence that some Treg also share some functional capabilities with Tconv, in particular the ability to produce proinflammatory cytokines. Tregand Th17-like cells have been linked in an evolutionary sense, because of their emergence at the same phylogenic stage. These two cell populations share some induction requirements, such as the need for TGF- $\beta$, and their balance may play a role in promoting healthy and diverse commensal colonisation, for example, in the gut (Ref. 64). Hence, several groups have tested whether Treg can be skewed towards IL17 production upon stimulation in vitro. Koenen et al. reported that IL-1 $\beta$, antigen-presenting cells and epigenetic modifications are necessary to induce IL-17 production by Treg (Ref. 65). Others showed that TLR2 stimulation may contribute to conversion of Treg to Th17-like cells with lower levels of FoxP3 expression and reduced suppressive capacity (Ref. 66). The authors suggested that this could represent an intermediate population, which is losing Treg function and become Th17-like Tconv. 
In vitro expansion and induction of Treg has been shown to induce the development of IFN$\gamma$-producing Treg (Ref. 67). In this study, the IFN- $\gamma$ frequency within FoxP3+ cells was enhanced by addition of IL-12 to the culturing conditions. These cytokine-producing induced Treg showed lower levels of demethylation at the TSDR region, suggesting that they may represent an intermediate cell population. Furthermore, samples from patients with type 1 diabetes showed an increased frequency of IFN- $\gamma+$ Treg after expansion, implicating a connection between inflammation and cytokineproducing Treg. Similarly, in a mouse model of colitis, an IFN- $\gamma$-producing inducible Treg subset has been observed interestingly, these IFN- $\gamma$ producing Treg were suppressive in vivo (Ref. 68). Recently, it has been shown that Treg isolated during the early inflammatory response following surgery were less suppressive than Treg isolated presurgery or at a later time point postsurgery despite remaining anergic (Ref. 69). This suggests that Treg in an acute early inflammatory response, such as after surgery, did not convert to full effector phenotype. Together, these studies suggest that Treg can produce cytokines, perhaps under the influence of proinflammatory conditions, yet remain functionally suppressive. The precise expansion or induction system used to generate these cytokine producing Treg may be crucial to their functionality. For example, Zheng et al. showed that the ratio of $B$ cells to $T$ cells was critical in yielding either functional Treg that produced cytokine or highly activated effector Tconv (Ref. 53).

Treg isolated from several chronic inflammatory or autoimmune conditions have been demonstrated to be functional in vitro. Cytokine-producing Treg are increased in inflammatory environments (Refs 68, 70, 71), but can also be found upon ex vivo stimulation of healthy peripheral blood mononuclear cells (PBMC) as a small proportion of total Treg (Refs 54, 72, 73, 74, 75). The frequency of cytokine-producing Treg can be increased by cytokine treatments. IL-17 production by Treg was enhanced by IL-6, IL-1 $\beta$, IL-21 alone or in various combinations. IL-23 alone had no effect, but could act synergistically in combination with the cytokines above (Refs 70, 73 , 74). The frequency of IFN- $\gamma+$ Treg was increased by IL-12 treatment in several studies (Refs 68, 71, 76). These data show that the polarisation 'requirements' for cytokine-producing Treg closely mirror those that drive polarisation of T effector cells.

The cytokine-producing Treg in these studies were anergic in vitro without the addition of IL2 , and were suppressive in Tconv co-cultures (Refs 54, 68, 70, 71, 72). In addition, the TSDR region was predominantly demethylated (Refs 71, 72, 76), and demonstrated a stable phenotype in long-term cultures or cloning (Refs 54, 71, 72, 73, 74), thus suggesting a bona fide Treg phenotype. We have recently demonstrated that the cytokine-producing Treg are contained within the CD161+ Treg population (Ref. 72). Cytokine-producing Treg also share expression of the transcription factors and, where tested, chemokine receptors, that are associated with expression of the respective cytokine in Tconv. Thus, IFN- $\gamma$-producing Treg have been shown to express Tbet (and CXCR3) and IL-17+ Treg express RORCv2 (and CCR6). Furthermore, Treg from mice lacking BCL6 show a Th2-Treg hybrid phenotype (Ref. 77) with higher levels of IL-4, IL-5, IL-13 transcripts and increased levels of GATA3.

A beneficial role in the protection against infection has been proposed for cytokineproducing Treg (Refs 54, 73, 76), with some evidence for specific expansion and cytokine production against certain pathogenic antigens (Refs 54, 76). Moreover, there is evidence that such Treg may play a beneficial role during transplantation (Refs 76,78 ): if IFN- $\gamma$ production is blocked or specifically knocked out in Treg in a transplant model, mice suffer from graftversus-host disease. Consequently, cytokineproducing Treg may represent an important mechanism during immune challenge, and their further investigation is warranted (Fig. 1).

\section{Biology of Treg in juvenile arthritis}

The paradox of increased Treg numbers in the inflammatory joint of JIA has been known for some years (Ref. 2). Interestingly, Treg in the blood of children with O-JIA appear to be present at normal or even decreased frequency, compared with healthy children (Refs 3, 79, 80). In an early study of the very severe form of JIA known as systemic JIA (sJIA; a form of disease where in addition to arthritis, patients display systemic symptoms such as fever, rash and major organ involvement), Treg were shown to be reduced in peripheral blood in severe active 
Figure 1. Regulatory T cell families. Schematic of four different Treg subfamilies (naïve, memory, activated memory and effector memory) with expression of CD4, CD25 and FoxP3 and suppressive function common to all, CD45RA expression defining naïve, and CD45RO memory Treg. Activated memory Treg also express activation markers such as GITR and CD62L, and potentially GARP and CD161. Effector memory Treg, in addition to being activated, also have the ability to produce pro-inflammatory cytokines like interleukin (IL)-17 or interferon gamma (IFN- $\mathrm{y}$ ), express specific cytokine and chemokine receptors (IL23R, IL1R, CCR6, CXCR3, etc) and additional transcription factors (Tbet, RORCv2, etc) to facilitate the partial Tconv-like phenotype.

disease, just before autologous stem cell transplantation. The frequency of Treg increased up to normal levels upon successful treatment by autologous stem cell transplantation (Ref. 81). However, a more recent study has suggested that while a difference in Treg frequency is observed between active and quiescent sJIA patients, Treg numbers in quiescent sJIA disease were raised compared with healthy controls as well as compared with active sJIA. In this study, the increase of total Treg, in the quiescent sJIA cases, appeared to be because of an increase in $\mathrm{CD} 45 \mathrm{RO}+$ memory Treg subpopulation, indicating that a rise in Treg occurs as disease resolution takes place (Ref. 82). It has recently been shown that the high level of inflammation in these patients, readily measureable in peripheral blood by the levels of the protein serum amyloid A (SAA) may directly affect Treg function, via the SAA protein itself, which prevents and reverses anergy in Treg without affecting their suppressive function (Ref. 83). Thus inflammatory proteins could drive an increase in Treg proliferation. 
We, and others, have shown a relationship of Treg frequency and disease severity in O-JIA. In the mild limited form of JIA known as persistent O-JIA the SF exudate has a higher frequency and absolute number of Treg compared with SF from children with the more severe extended $\mathrm{O}$ JIA (Refs 2, 3). These data suggest that although immune tolerance has been impaired in JIA, Treg frequency does correlate with disease severity and thus Treg might actively restrain inflammation. When Treg in the joint were compared with other T cell types within the SF, it was demonstrated that Treg frequency negatively correlates with Th17 frequency (Ref. 3). The same correlation has been observed for their respective transcription factors FoxP3 and RORCv2 (Th17) at the mRNA level (Ref. 80). Since Treg and Th17 have been associated in an evolutionary sense (Ref. 64), the balance between Treg and Th17 might play a crucial role in JIA, and local factors that affect this balance may correlate directly with the clinical course and outcome. In a study, which aimed to identify predictive biomarkers of extension to more severe disease early in O-JIA, we observed that this difference in Treg frequency was present very early, even before extension, with lower Treg frequency in children destined to go on to more severe arthritis (so-called extendedto-be O-JIA) (Ref. 84). Interestingly, RORC2 was one of the gene transcripts found to be significantly differentially expressed between patients with persistent O-JIA and extended-to-be O-JIA phenotype (Ref. 84).

Since Treg within the joint clearly do not resolve inflammation, many studies examining their phenotype and function have been performed to determine if there is an intrinsic defect in the Treg of JIA patients. Treg found in the inflamed joint all have a memory phenotype $(\mathrm{CD} 45 \mathrm{RO}+)$ (Ref. 2) and are activated, as demonstrated by high HLA-DR and GITR expression. Expression of CTLA4 and CD39 is also highly enriched on SF Treg (Refs 2, 47). Interestingly, in the SF of JIA, in addition to CD39 on Treg, a population of CD39 + FoxP3 - cells has been demonstrated. Both Treg and non-Treg expressing CD39 are functional in breaking down ATP (Ref. 47).

One hallmark of Treg function is the ability to suppress Tconv in vitro. Several reports have reported potent suppressive capacity of SF Treg (Refs 2, 47, 85, 86, 87). However, more recently, there have been studies demonstrating that SF
Treg fail to suppress Tconv from the joint but can suppress blood Tconv (Refs 4, 5). Together, these studies suggest that Treg from patients with JIA do not appear to have an intrinsic defect, as they function well when taken out of their natural environment. However, factors in the local microenvironment in the joint, or the effector $\mathrm{T}$ cells themselves, may affect the function of regulatory cells in a way that they cannot control inflammation within the joint.

Cytokines can abrogate suppression by Treg by either affecting Treg directly or effector T cells. IL-2, IL-6, IL-7, IL-15 and tumour necrosis factor alpha (TNF- $\alpha$ ) have all been implicated in diminishing the suppressive capacity of Treg and these cytokines are present at high levels in the SF of JIA (Refs $4,85,88$ ). Both TNF- $\alpha$ and IL- 6 are now successfully targeted by therapeutic approaches in some JIA patients. IL- 6 and TNF- $\alpha$ have been shown to induce higher phosphorylation levels of protein kinase $\mathrm{B}$, thus activating signaling in effector T cells. This, in turn, renders effector $\mathrm{T}$ cells unresponsive to both TGF- $\beta$ and Treg mediated suppression (Ref. 4). Synovial effector T cells have also been shown to be able to switch from IL-17 to IFN- $\gamma$-producing cells in the environment of the joint and in vitro (Refs 89, 90). The major cytokine responsible for this plasticity is IL-12, which is also enriched within the joint. The addition of TGF- $\beta$ could inhibit this conversion to some extent, but the measurements showed low levels of TGF- $\beta$ in SF. Given that SF Tconv are strong producers of pro-inflammatory cytokines it is plausible that locally these effector $\mathrm{T}$ cells may contribute to the failure of regulation by Treg (Ref. 5).

The recent descriptions of pro-inflammatory cytokine-producing Treg in other autoimmune conditions led us to investigate whether JIA Treg also demonstrate this phenomenon. We found a clear enrichment of Treg with a pro-inflammatory potential (enrichment for pro-inflammatory cytokine production ex vivo and in vitro), defined by CD161 expression, a factor that may also contribute to the apparent 'failure' by Treg to suppress ongoing inflammation in JIA (Ref. 72).

In addition to these phenotypic and functional data, there is evidence that genetic factors influence Treg biology in JIA. Genetic associations with the IL2 and IL10 gene regions and the gene encoding CD25 (the IL-2R $\alpha$-chain) have been demonstrated in JIA (Refs 91, 92, 93, 94); these genes are important in Treg maintenance and function. 
Several of these associations have recently been confirmed at genome-wide association level of significance (Ref. 95). However, no genetic association with any FOXP3 SNP itself, or CTLA4, could be found (Refs 96, 97).

Collectively, these data suggest that the inflammatory environment plays an important role in determining the overall effectiveness of regulatory cells at the diseased site (Fig. 2). The main findings on Treg in JIA are summarised in Table 1. Thus, cytokines and other inflammatory mediators may render $\mathrm{T}$ effector cells unresponsive to suppression. However, Treg should not be disregarded since they clearly have an effect upon the clinical outcomes and their manipulation may be possible in the future, to restore full tolerance. With conflicting reports on Treg function, and the emerging knowledge of their capacity of making pro-inflammatory cytokines themselves, it is crucial to investigate how these different Treg populations act at the site of inflammation, how stable they are and what the effects of the local environment may be on Treg behaviour and phenotype.

\section{Clinical implications}

Regulatory $\mathrm{T}$ cells could potentially be used as cellular therapy themselves for autoimmune disease. In addition, many treatments will affect Treg balance and function. Animal models have been useful in assessing the role of Treg in treatment of arthritis.

\section{Antigen specificity of Treg}

One key issue is whether Treg influence disease in an antigen-specific or non-specific manner. Many arthritis models in rodents use antigen to induce arthritis. Antigen-specific induced Treg and natural Treg can prevent disease in these model systems, but natural Treg did not reverse established disease (Refs 32, 98), whereas the use of induced Treg reduced disease severity and incidence rate slightly in some cases (Ref. 32). The depletion of Treg before disease initiation has been shown to worsen the disease in transgenic, known antigen-driven and spontaneous models (Refs 98, 99, 100). In some models, the specific antigen recognised by Treg that can modulate disease is unknown or unclear; However, considerable evidence from several groups suggests that Treg do require a TCR/antigen signal for them to elicit effective suppression (Ref. 101).
To address the importance of antigen specific Treg action, $\mathrm{Oh}$ et al. used a transgenic mouse model with Treg and effector $\mathrm{T}$ cells of mixed specificity and an auto-antigen linked to MHC class II (Ref. 102). These mice develop spontaneous arthritis, which is driven by IL-17. Transfer of Treg that were enriched for autoantigen specificity could not prevent disease or perform bystander suppression, even though they were functional towards the same antigenspecific effector cells in vitro, and in a separate in vivo model. Interestingly, polyclonal Treg did prevent disease, indicating that a mixed population of Treg, presumably with multiple antigen specificities, different to the disease driving effector cell specificity, may be effective in arthritis control.

By using a different transgenic model, CD4T cells expressing a TCR against a cutaneous selfantigen (SFZ70) resulted in severe lymphocytic infiltration of the skin and liver in Treg-deficient animals, whereas in non-deficient mice only a few developed caudal dermatitis $(<2 \%)$ with a high frequency of FoxP3+ among CD4+ T cells. A comparison of the effector $\mathrm{T}$ cells between the two groups revealed that the antigen-specific Treg actively suppressed expression of skin homing receptors and cytokine production by effector T cells (Ref. 99). Similarly, in a human graft-versus-host disease skin explant model early Treg treatment inhibited tissue infiltration of primed CD8T cell by downregulation of their homing receptors, such as CXCR3 and cutaneous leucocyte-associated antigen, and an overall decrease in chemokine production (Ref. 103). Therefore, Treg might prevent homing to the site of inflammation by inhibiting the expression of homing receptors on effector cells. In JIA inflammation is highly localised to the affected joints. Therefore, if Treg could inhibit homing of inflammatory cells to the joint, this might present an opportunity to stop disease progression.

In patients with JIA, stimulation of T cells with a human auto-antigen (Hsp60) results in different responses depending on disease severity (Refs 104, 105). Surprisingly active O-JIA PBMC show high IL-10/IFN- $\gamma$ ratios, whereas samples from patients in remission had high levels of IFN- $\gamma$, thus a low IL- $10 /$ IFN- $\gamma$ ratio. Polyarticular JIA samples showed no difference between active disease and remission with overall low IL-10/IFN- $\gamma$ ratios. These data 

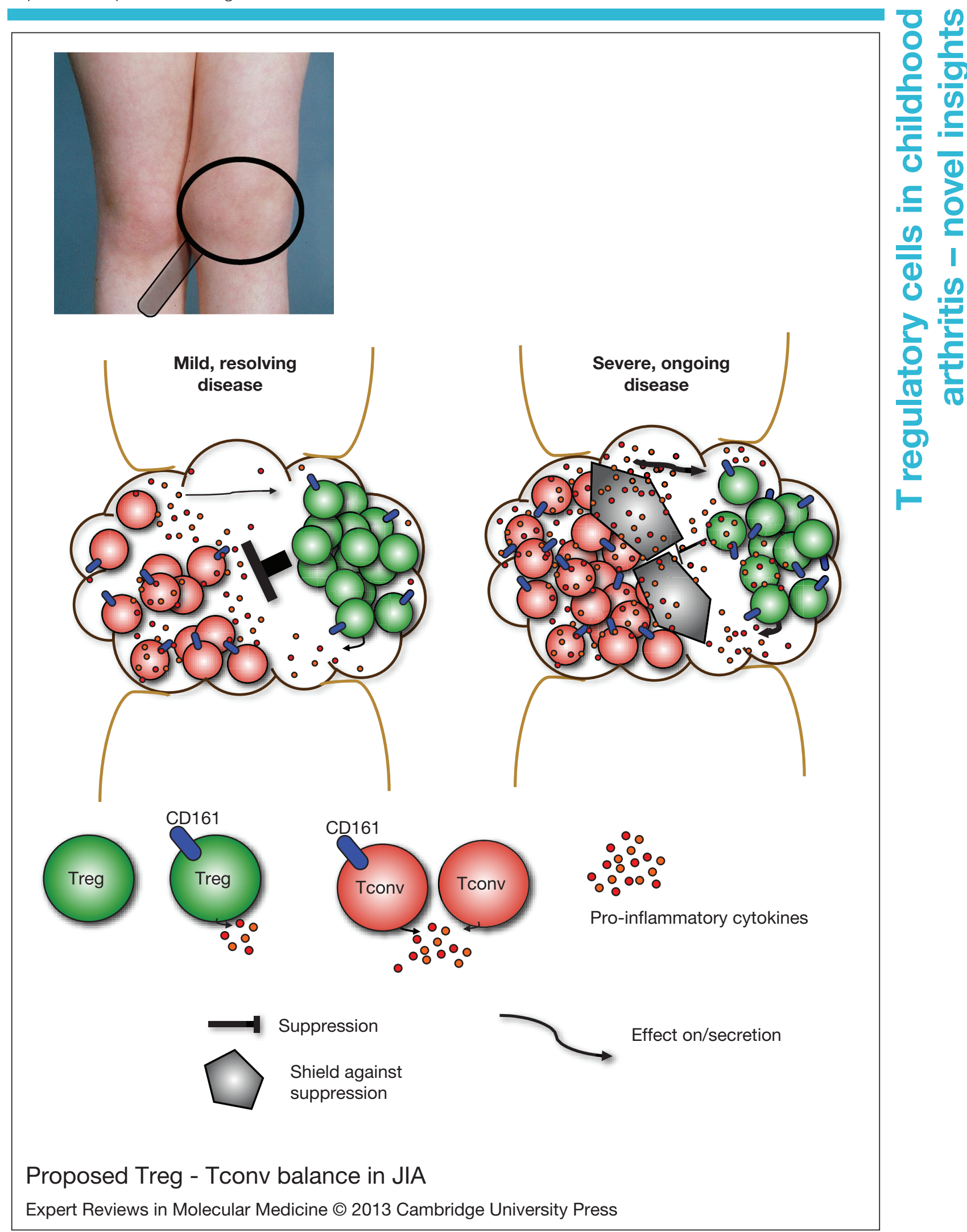

Figure 2. Proposed Treg-Tconv balance in juvenile idiopathic arthritis (JIA). (See next page for legend.)

Accession information: doi:10.1017/erm.2013.14; Vol. 15; e13; November 2013

(c) Cambridge University Press 2013. The online version of this article is published within an Open Access environment subject to the conditions of the Creative Commons Attribution licence http://creativecommons.org/licenses/by/3.0/. 
suggest that responses to self-hsp60 antigen, and in particular an enrichment of Treg that are specific for hsp60 antigens, correlate with good outcomes in JIA (Refs 104, 106).

\section{Influencing Treg in vivo}

Strategies aiming to influence Treg function in vivo by either increasing the number or efficiency of Treg could be a valuable treatment option. Small biologically active molecules have been used to expand mice, rat and human Treg with increased suppressive capacity in vitro and in vivo that have been used for the treatment of animal models of disease (Refs 107, 108, 109, $110,111)$. We have studied vasoactive intestinal peptide (VIP), a small neuro-peptide with immunoregulatory properties. Effects of VIP and its receptors can induce tolerance or abrogate inflammation in autoimmune models (Refs 108, 112, 113, 114, 115, 116, 117). Thus, for example, VIP prevents and treats collagen-induced arthritis in rodents directly, or by transfer of Treg that were VIP-treated in vitro or isolated from VIP-treated mice (Refs 108, 118). Lentiviral delivery of the VIP gene into mice with collagen-induced arthritis resulted in complete regression of disease, which was associated with an increase in FoxP3+CD25+ Treg (Ref. 119). Besides the direct effect of VIP on $\mathrm{T}$ cells (Refs 108, 120, 121), VIP also induces tolerogenic dendritic cells (DC), which in turn induce Treg (Refs 113, 114, 122, 123). The anti-inflammatory actions of VIP require interaction with VIP receptor 1 (VIPR1) (Refs 109, 112, 121). Beneficial effects of VIP and VIPR1 agonists have also been reported for human cells (Refs 109, 121, 123). Furthermore, VIPR1 expression is decreased in some autoimmune diseases (Refs 124, 125, 126), including JIA (Ref. 127), suggesting that the lack of VIP-VIPR1 signaling might be involved in disease pathogenesis. Improved delivery methods and modifications of agents to increase half-life are showing promising results in in vitro and animal models (Refs 114, 119, 128, 129) and demonstrate the potential of small molecules as treatment strategies.

\section{Monoclonal antibody therapy}

In recent years, monoclonal antibody treatments that modulate the immune system have been very successful, both in animal models and in the clinic. These treatments may affect Treg directly or indirectly. As described above, Treg exhibit some of their regulatory capacity through CTLA4. CTLA4-deficient mice develop spontaneous and fatal systemic autoimmune disease and have less functional Treg (Ref. 130). In patients with rheumatoid arthritis (RA), a defect in CTLA4 expression and recycling resulting in less functional Treg, because of decreased signaling through CTLA4, has been demonstrated; this could be reversed in vitro by chemically resurfacing CTLA4 (Ref. 131). These results are intriguing given the availability of a therapeutic agent, which is recombinant CTLA4-antibody complex (CTLA4-Ig: including Abatacept and Belatacept), for the treatment of autoimmune disease. CTLA4 is a ligand for co-stimulatory molecules CD80 and CD86, expressed on professional antigen-presenting cells, including DC and B cells, and competes efficiently with CD28 for binding to these molecules. The CTLA4Ig fusion drug is thought to exert its therapeutic effects through its high affinity binding to CD80/ 86, thus blocking $\mathrm{T}$ cell activation. However, evidence also exists to suggest that its effects may also alter Treg function. In mouse models of collagen-induced arthritis, CTLA4-Ig treatment decreases disease severity (Ref. 132). Interestingly, the drug alters DC, which become tolerogenic DC, and induces highly suppressive Treg. CTLA4-Ig therapy is successfully used in RA (Refs 133, 134) and JIA (Ref. 135). It has been reported that in patients with RA, Abatacept led to a reduction in peripheral blood Treg numbers and a significant enhancement in Treg function on a per cell basis (Ref. 136).

Figure 2. Proposed Treg-Tconv balance in juvenile idiopathic arthritis (JIA). (Legend; see previous page for figure.) Schematic of milder resolving (left) and more severe and ongoing (right) inflammation of the joint, showing infiltration of cells. Treg are highlighted in green, Tconv in red, with CD161 expression in blue. Proinflammatory cytokines are depicted as small red and orange circles. In mild, resolving disease, more Treg are present in the joint than in severe ongoing disease. We hypothesise that in mild, resolving inflammation Tconv may be more susceptible to suppression (big block bar), and inflammatory effects of the micro-environment are smaller (thin arrows) than in more severe disease. In addition, a higher frequency of CD161+ Treg appears to associate with more severe disease. 
Table 1. Evidence for the role or dysfunction of Treg in JIA

\section{Key findings}

High Treg frequency in the inflamed joint

Treg frequency normal in blood

Low Treg frequency in blood of active severe cases, before autologous stem cell transplantation

Treg frequency in blood decreased in active vs. quiescent disease, but raised memory Treg frequency in quiescent versus healthy cases

Serum amyloid (SAA) protein is high. SAA breaks Treg anergy without affecting their suppressive function

Increased Treg frequency in the milder persistent O-JIA compared with the more severe extended O-JIA and compared with extended-to-be O-JIA (before extension has occurred)

Reciprocal relationship between Treg frequency/FoxP3 transcripts and Th17 frequency/RORCv2 transcripts

Treg in the inflamed joint have an activated memory phenotype (high expression of: CD45RO, GITR, HLA-DR, CTLA4 and CD39)

In the SF increase of CD39 + FoxP3+ Treg and CD39 + FoxP3 - Tcells; CD39 is functional on both subsets in vitro

SF Treg have potent suppressive behaviour in vitro

SF Treg do not suppress SF Tconv, but do suppress blood Tconv

Cytokines that can affect Treg suppressive capacity are increased in SF

CD161 + SF Tconv can change their cytokine profile (IL-17 to IL-17 and IFN- $\gamma$ double to IFN- $\gamma$ single producers) in vitro and in vivo

CD161+ Treg are increased in the inflamed joint. CD161+ Treg can produce pro-inflammatory cytokines (IL-17, IFN-Y and IL-2)

Genetic associations can be found in JIA with genes important for Treg function (including IL2, IL10 and CD25); several have been confirmed on a genome wide association level

No genetic association with FOXP3 or CTLA4 has been found

Self-antigen (Hsp60) specific Treg can be associated with remission of O-JIA (but not in P-JIA)

\section{Disease/JIA subtype*}

All JIA

O-JIA, P-JIA and SJIA

SJIA

SJIA

82

SJIA

O-JIA

$2,3,84$

O-JIA

3, 80

O-JIA

2, 47

O-JIA

47

O-JIA, P-JIA, sJIA, psJIA and RA

$2,47,85,86$ 87

O-JIA and P-JIA

4,5

O-JIA, P-JIA and SJIA

4, 85, 88

O-JIA

89,90

O-JIA and P-JIA

72

all JIA

91, 92, 93, 94, 95

all JIA

96, 97

O-JIA and P-JIA

*Definitions according to Petty et al. (Ref. 1); O-JIA, oligoarticular JIA (juvenile idiopathic arthritis); P-JIA, polyarticular JIA; psJIA, psoriatic JIA; RA, rheumatoid arthritis; SF, synovial fluid; sJIA, systemic JIA; Tconv, conventional T cells.

Accession information: doi:10.1017/erm.2013.14; Vol. 15; e13; November 2013 environment subject to the conditions of the Creative Commons Attribution licence http://creativecommons.org/licenses/by/3.0/. 
For some novel therapeutic agents, results in murine models and humans have been specifically different. For example, use of the second-generation co-stimulation-blocking molecule, Belatacept, in renal transplant patients, has been shown to lead to an increase in FoxP3+ Treg cells in the allograft (Ref. 137). This was notably different to the results in mice, where CTLA4-Ig has been shown to compromise Treg function (Refs 138, 139). These results, as well as the near disastrous results of blockade of CD28 in a phase I trial of a super agonist anti-CD28 antibody, TGN1412, emphasise that extrapolation from the rodent to the human immune system is not always wise (Ref. 140).

TNF- $\alpha$ has been implicated in the pathogenesis of many forms of human inflammatory arthritis, including JIA, where it has been shown to be highly enriched within the inflammatory joint (Ref. 88). There are several TNF- $\alpha$ blockade agents now being used to treat JIA. Infliximab and Adalimumab are both anti-TNF- $\alpha$ antibodies, whereas Etanercept is a soluble TNF-receptor fusion protein. A third concept is TNF-kinoids, which are hybrid molecules used to vaccinate the recipient, such as TNF conjugated to a carrier protein (e.g. keyhole limpet haemocyanin) that induces production of polyclonal self-antibodies against TNF by the host. Mice with knock-in of the TNF- $\alpha$ gene develop arthritis, and Treg at the peak of this disease model show only weak suppressive capacity (Ref. 141). Treatment with anti-TNF- $\alpha$ (Infliximab) or a TNF- $\alpha$-kinoid ameliorated disease and increased Treg frequency and function. In vitro treatment of healthy human Treg with TNF- $\alpha$ rendered them less functional and decreased their level of FoxP3, mimicking the phenotype of Treg from RA patients. Treg function and FoxP3 could be restored by culture with anti-TNF- $\alpha$ antibodies. Treatment with Infliximab in RA patients can restore Treg levels and function and the population of Treg that develop upon Infliximab treatment has been shown to be a novel, induced CD62L- Treg population (Refs 142, 143). Others have suggested that Treg from RA patients actually have a high amount of TNF- $\alpha$ bound to their surface membrane, which affects their functionality (Ref. 144).

TNF-blockade alone is not as efficient in treating arthritis compared with combinational therapy with methotrexate (MTX) (Ref. 145). This synergy between MTX and anti-TNF- $\alpha$ treatment is also clear for both RA and JIA (Refs 135, 146, 147). A placebo-controlled study in RA showed a slightly higher increase in Treg with anti-TNF- $\alpha$ plus MTX, compared with the placebo plus MTX group (Ref. 148). In a study showing significant improvement of RA patients on Etanercept plus MTX, where the MTX alone group showed only a trend towards improvement, the ratio between Treg to Th17 was seen to increase after treatment (Ref. 149). Others, however, did not find any prominent difference in the Treg/Th17 ratio between MTX alone or in combination with either Etanercept or Adalimumab (Refs 150, 151). A recent study, exploring the mechanism of Treg in Adalimumab-responding RA patients compared with non-responders and active RA, found that Treg isolated from Adalimumabresponding patients were able to suppress IL-17 production in vitro, acting via the inhibition of monocyte-derived IL-6, whereas non-responders or active RA samples did not (Ref. 152). Interestingly, this study also compared Treg from Etanercept-treated patients, which did not suppress IL-17 in vitro independent of clinical response to the drug. Therefore, different TNF- $\alpha$ blockade approaches seem to have different effects on the Treg and Th17 balance.

Although biologics have drastically improved treatment for many RA and JIA patients, not all patients respond in a similar way; these drugs are relatively expensive and, to date, are not widely available in all countries. Further characterisation and definition of different subgroups of the disease will therefore be crucial to develop biomarkers that can be used to help predict which treatment will be best for each patient.

\section{Cellular therapies}

As mentioned previously, Treg cellular adoptive transfer has been used successfully to treat experimental arthritis. However, this approach poses a variety of problems. One concern is that some Treg could themselves add further to the inflammatory response by switching on the production of cytokines, as described above. It would therefore be useful to develop a strategy to ensure that Treg with pro-inflammatory properties are not transferred or at least could be monitored (Ref. 72). Others have used the approach to initiate activation-induced cell death during Treg expansion, which should 
eliminate proinflammatory cells close to the end of their lifespan (Ref. 153). However, it is still unclear as to what might happen to expanded or induced Treg transferred into an inflammatory environment. Several animal models showed positive outcomes when used in the prevention of disease, including antigeninduced arthritis (Ref. 98), but most Treg adoptive-transfer models could not reverse established disease. It is also known that if Treg alone are transferred into an "empty mouse", without other adaptive immune cells, a large proportion will lose their Treg status as a result of a lack of IL-2 which is needed for survival (Ref. 154). Therefore, maybe a transplant of stem cells, which can reestablish all the cells of the immune system, might be more favourable. To reset and reconstitute the immune system, autologous stem cell transplantation (ASCT) has successfully been used in the treatment of severe systemic and polyarticular cases of JIA (Refs 81, 155, 156, 157, 158). After ASCT, Treg numbers increased relative to $\mathrm{CD} 4+\mathrm{T}$ cells as a whole, suggesting a resetting of the immune system. However, it has been established that adequate ablation of the immune system before transplantation is essential to prevent early relapse of the disease in this approach.

In addition to Treg, human mesenchymal stem cells (MSC) offer a new treatment avenue (Ref. 159). MSC can actively suppress immune responses (Ref. 160). In a model system, human MSC have successfully been used to treat experimental arthritis in mice (Ref. 161). In this study, the development of antigen-specific mouse Treg was induced by the human MSC and were essential for amelioration of disease.

\section{Conclusions}

Increasing our knowledge of the fundamental biology of Treg will be extremely important in helping to optimise the clinical use of adoptive Treg. Current approaches that use and monitor Treg are already being tested in clinical trials: Treg are being used to improve transplant survival and reduction of graft-versus-host disease, and initial trials for Treg as cellular therapy of type one diabetes are also underway. These trials are mainly at the safety and efficiency testing stage, using different dosing regimes and variable immunosuppression. It will be exciting to follow these and other ongoing trials; their outcomes might point us towards novel ways of treating autoimmunity and will allow us to monitor the safety and behaviour of human Treg that have been adoptively transferred into inflammatory environments.

\section{Acknowledgements and funding}

The authors thank the group members for their help and advice and C Duurland for careful reading of the manuscript. LRW is partly supported by funding from Great Ormond Street Children's Charity and the National Institute for Health research (NIHR), UK. AMP was supported by the Nuffield Oliver Bird Programme. The authors declare no competing financial interests.

\section{References}

1 Petty, R.E. et al. (2004) International League of Associations for Rheumatology classification of juvenile idiopathic arthritis: second revision, Edmonton, 2001. Journal of Rheumatology 31, 390-392

2 de Kleer, I.M. et al. (2004) CD4 + CD25 bright regulatory $\mathrm{T}$ cells actively regulate inflammation in the joints of patients with the remitting form of juvenile idiopathic arthritis. Journal of Immunology 172, 6435-6443

3 Nistala, K. et al. (2008) Interleukin-17-producing T cells are enriched in the joints of children with arthritis, but have a reciprocal relationship to regulatory T cell numbers. Arthritis \& Rheumatism 58, 875-887

4 Wehrens, E.J. et al. (2011) Functional human regulatory $\mathrm{T}$ cells fail to control autoimmune inflammation due to $\mathrm{PKB} / \mathrm{c}$-akt hyperactivation in effector cells. Blood 118, 3538-3548

5 Haufe, S. et al. (2011) Impaired suppression of synovial fluid CD4 + CD25- T cells from patients with juvenile idiopathic arthritis by CD4 + CD25+ Treg cells. Arthritis \& Rheumatism 63, 3153-3162

6 Sakaguchi, S. et al. (1995) Immunologic selftolerance maintained by activated T cells expressing IL-2 receptor alpha-chains (CD25). Breakdown of a single mechanism of self-tolerance causes various autoimmune diseases. Journal of Immunology 155, 1151-1164

7 Stephens, L.A. and Mason, D. (2000) CD25 is a marker for CD4+ thymocytes that prevent autoimmune diabetes in rats, but peripheral $\mathrm{T}$ cells with this function are found in both CD25+ and CD25- subpopulations. Journal of Immunology $165,3105-3110$

8 Thornton, A.M. and Shevach, E.M. (1998) CD4 + CD25+ immunoregulatory T cells suppress 
polyclonal $\mathrm{T}$ cell activation in vitro by inhibiting interleukin 2 production. Journal of Experimental Medicine 188, 287-296

9 Gavin, M.A. et al. (2002) Homeostasis and anergy of CD4(+)CD25(+) suppressor T cells in vivo. Nature Immunology 3, 33-41

$10 \mathrm{Ng}$, W.F. et al. (2001) Human CD4(+)CD25(+) cells: a naturally occurring population of regulatory $\mathrm{T}$ cells. Blood 98, 2736-2744

11 Baecher-Allan, C., Viglietta, V. and Hafler, D.A. (2002) Inhibition of human CD4(+)CD25(+high) regulatory $\mathrm{T}$ cell function. Journal of Immunology 169, 6210-6217

12 Dieckmann, D. et al. (2001) Ex vivo isolation and characterization of CD4(+)CD25(+) T cells with regulatory properties from human blood. Journal of Experimental Medicine 193, 1303-1310

13 Stephens, L.A. et al. (2001) Human CD4(+)CD25(+) thymocytes and peripheral T cells have immune suppressive activity in vitro. European Journal of Immunology 31, 1247-1254

14 Levings, M.K. et al. (2002) Human CD25 + CD4+ T suppressor cell clones produce transforming growth factor beta, but not interleukin 10, and are distinct from type $1 \mathrm{~T}$ regulatory cells. Journal of Experimental Medicine 196, 1335-1346

15 Brunkow, M.E. et al. (2001) Disruption of a new forkhead/winged-helix protein, scurfin, results in the fatal lymphoproliferative disorder of the scurfy mouse. Nature Genetics 27, 68-73

16 Wildin, R.S., Smyk-Pearson, S. and Filipovich, A.H. (2002) Clinical and molecular features of the immunodysregulation, polyendocrinopathy, enteropathy, X linked (IPEX) syndrome. Journal of Medical Genetics 39, 537-545

17 Hori, S., Nomura, T. and Sakaguchi, S. (2003) Control of regulatory $\mathrm{T}$ cell development by the transcription factor Foxp3. Science 299, 1057-1061

18 Fontenot, J.D., Gavin, M.A. and Rudensky, A.Y. (2003) Foxp3 programs the development and function of CD4 + CD25+ regulatory T cells. Nature Immunology 4, 330-336

19 Khattri, R. et al. (2003) An essential role for Scurfin in CD4 + CD25+ T regulatory cells. Nature Immunology 4, 337-342

20 Yagi, H. et al. (2004) Crucial role of FOXP3 in the development and function of human CD25 + CD4+ regulatory $\mathrm{T}$ cells. International Immunology 16, 1643-1656

21 Law, J.P. et al. (2009) The importance of Foxp3 antibody and fixation/permeabilization buffer combinations in identifying CD4 + CD25 + Foxp3+ regulatory T cells. Cytometry A 75, 1040-1050
22 Baron, U. et al. (2007) DNA demethylation in the human FOXP3 locus discriminates regulatory $\mathrm{T}$ cells from activated FOXP3(+) conventional T cells. European Journal of Immunology 37, 2378-2389

23 Floess, S. et al. (2007) Epigenetic control of the foxp3 locus in regulatory T cells. PLoS Biology 5, e38

24 Polansky, J.K. et al. (2008) DNA methylation controls Foxp3 gene expression. European Journal of Immunology 38, 1654-1663

25 Thornton, A.M. et al. (2010) Expression of Helios, an Ikaros transcription factor family member, differentiates thymic-derived from peripherally induced Foxp3 + T regulatory cells. Journal of Immunology 184, 3433-3441

26 Verhagen, J. and Wraith, D.C. (2010) Comment on "Expression of Helios, an Ikaros transcription factor family member, differentiates thymic-derived from peripherally induced Foxp3+ T regulatory cells". Journal of Immunology 185, 7129; author reply 30

27 Gottschalk, R.A., Corse, E. and Allison, J.P. (2012) Expression of Helios in peripherally induced Foxp3+ regulatory T cells. Journal of Immunology 188, 976-980

28 Zheng, Y. et al. (2010) Role of conserved non-coding DNA elements in the Foxp3 gene in regulatory Tcell fate. Nature 463, 808-812

29 Haribhai, D. et al. (2011) A requisite role for induced regulatory $\mathrm{T}$ cells in tolerance based on expanding antigen receptor diversity. Immunity 35, 109-122

30 Hippen, K.L. et al. (2011) Generation and large-scale expansion of human inducible regulatory $\mathrm{T}$ cells that suppress graft-versus-host disease. American Journal of Transplantation 11, 1148-1157

31 Beres, A. et al. (2011) Instability of Foxp3 expression limits the ability of induced regulatory $\mathrm{T}$ cells to mitigate graft versus host disease. Clinical Cancer Research 17, 3969-3983

32 Kong, N. et al. (2012) Antigen-specific transforming growth factor beta-induced Treg cells, but not natural Treg cells, ameliorate autoimmune arthritis in mice by shifting the Th17/Treg cell balance from Th17 predominance to Treg cell predominance. Arthritis \& Rheumatism 64, 2548-2558

33 Evans, R.L. et al. (1978) Peripheral human T cells sensitized in mixed leukocyte culture synthesize and express Ia-like antigens. Journal of Experimental Medicine 148, 1440-1445

34 Ko, H.S. et al. (1979) Ia determinants on stimulated human T lymphocytes. Occurrence on mitogenand antigen-activated $\mathrm{T}$ cells. Journal of Experimental Medicine 150, 246-255 
35 Baecher-Allan, C. et al. (2001) CD4 + CD25 $5^{\text {high }}$ regulatory cells in human peripheral blood. Journal of Immunology 167, 1245-1253

36 Baecher-Allan, C., Wolf, E. and Hafler, D.A. (2006) MHC class II expression identifies functionally distinct human regulatory T cells. Journal of Immunology 176, 4622-4631

37 Kleinewietfeld, M. et al. (2005) CCR6 expression defines regulatory effector/memory-like cells within the CD25(+)CD4+ T-cell subset. Blood 105, 2877-2886

38 Miyara, M. et al. (2009) Functional delineation and differentiation dynamics of human CD4+ T cells expressing the FoxP3 transcription factor. Immunity 30, 899-911

39 Booth, N.J. et al. (2010) Different proliferative potential and migratory characteristics of human $\mathrm{CD} 4+$ regulatory $\mathrm{T}$ cells that express either CD45RA or CD45RO. Journal of Immunology 184, 4317-4326

40 Vukmanovic-Stejic, M. et al. (2006) Human CD4+ CD25hi Foxp3+ regulatory $\mathrm{T}$ cells are derived by rapid turnover of memory populations in vivo. Journa of Clinical Investigation 116, 2423-2433

41 Takahashi, T. et al. (2000) Immunologic selftolerance maintained by CD25(+)CD4(+) regulatory $\mathrm{T}$ cells constitutively expressing cytotoxic T lymphocyte-associated antigen 4. Journal of Experimental Medicine 192, 303-310

42 Deaglio, S. et al. (2007) Adenosine generation catalyzed by CD39 and CD73 expressed on regulatory $\mathrm{T}$ cells mediates immune suppression. Journal of Experimental Medicine 204, 1257-1265

43 Borsellino, G. et al. (2007) Expression of ectonucleotidase CD39 by Foxp3+ Treg cells: hydrolysis of extracellular ATP and immune suppression. Blood 110, 1225-1232

44 Walker, L.S. and Sansom, D.M. (2011) The emerging role of CTLA4 as a cell-extrinsic regulator of T cell responses. Nature Reviews Immunology 11, 852-863

45 Dwyer, K.M. et al. (2007) CD39 and control of cellular immune responses. Purinergic Signal 3, $171-180$

46 Mandapathil, M. et al. (2010) Generation and accumulation of immunosuppressive adenosine by human CD4 + CD25 ${ }^{\text {high }} \mathrm{FOXP3}+$ regulatory T cells. Journal of Biological Chemistry 285, 7176-7186

47 Moncrieffe, H. et al. (2010) High expression of the ectonucleotidase CD39 on T cells from the inflamed site identifies two distinct populations, one regulatory and one memory $\mathrm{T}$ cell population. Journal of Immunology 185, 134-143
48 Yamazaki, T. et al. (2008) CCR6 regulates the migration of inflammatory and regulatory $\mathrm{T}$ cells. Journal of Immunology 181, 8391-8401

49 Turner, J.E. et al. (2010) CCR6 recruits regulatory T cells and Th17 cells to the kidney in glomerulonephritis. Journal of the American Society of Nephrology 21, 974-985

50 Lim, H.W. et al. (2008) Human Th17 cells share major trafficking receptors with both polarized effector T cells and FOXP3+ regulatory T cells. Journal of Immunology 180, 122-129

51 Tosello, V. et al. (2008) Differential expression of CCR7 defines two distinct subsets of human memory CD4 + CD25+ Tregs. Clinical Immunology 126, 291-302

52 Koch, M.A. et al. (2009) The transcription factor T-bet controls regulatory $\mathrm{T}$ cell homeostasis and function during type 1 inflammation. Nature Immunology 10, 595-602

53 Zheng, J. et al. (2011) Generation of human Th1-like regulatory CD4+ T cells by an intrinsic IFNgamma- and T-bet-dependent pathway. European Journal of Immunology 41, 128-139

54 Duhen, T. et al. (2012) Functionally distinct subsets of human FOXP3 + Treg cells that phenotypically mirror effector Th cells. Blood 119, 4430-4440

55 Wang, R. et al. (2009) Expression of GARP selectively identifies activated human FOXP3+ regulatory T cells. Proceedings of the National Academic Sciences of the United States of America 106, 13439-13444

56 Wang, R. et al. (2008) Identification of a regulatory $\mathrm{T}$ cell specific cell surface molecule that mediates suppressive signals and induces Foxp3 expression. PLoS One 3, e2705

57 Stockis, J. et al. (2009) Membrane protein GARP is a receptor for latent TGF-beta on the surface of activated human Treg. European Journal of Immunology 39, 3315-3322

58 Kehrmann, J. et al. (2011) FOXP3 expression in GARP-transduced helper T cells is not associated with FOXP3 TSDR demethylation. Transfusion Medicine and Hemotherapy 38, 287-291

59 Zheng, Y. et al. (2009) Regulatory T-cell suppressor program co-opts transcription factor IRF4 to control $\mathrm{T}(\mathrm{H}) 2$ responses. Nature 458, 351-356

60 Chaudhry, A. et al. (2009) CD4+ regulatory T cells control TH17 responses in a Stat3-dependent manner. Science 326, 986-991

61 Chaudhry, A. et al. (2011) Interleukin-10 signaling in regulatory $\mathrm{T}$ cells is required for suppression of Th17 cell-mediated inflammation. Immunity 34, $566-578$ 
62 Chung, Y. et al. (2011) Follicular regulatory T cells expressing Foxp3 and Bcl-6 suppress germinal center reactions. Nature Medicine 17, 983-988

63 Linterman, M.A. et al. (2011) Foxp3(+) follicular regulatory $\mathrm{T}$ cells control the germinal center response. Nature Medicine 17, 975-982

64 Weaver, C.T. and Hatton, R.D. (2009) Interplay between the TH17 and TReg cell lineages: a (co-)evolutionary perspective. Nature Reviews of Immunology 9, 883-889

65 Koenen, H.J. et al. (2008) Human CD25 $5^{\text {high }}$ Foxp3pos regulatory $\mathrm{T}$ cells differentiate into IL-17-producing cells. Blood 112, 2340-2352

66 Nyirenda, M.H. et al. (2011) TLR2 stimulation drives human naive and effector regulatory $\mathrm{T}$ cells into a Th17-like phenotype with reduced suppressive function. Journal of Immunology 187, 2278-2290

67 McClymont, S.A. et al. (2011) Plasticity of human regulatory $\mathrm{T}$ cells in healthy subjects and patients with type 1 diabetes. Journal of Immunology 186, 3918-3926

68 Feng, T. et al. (2011) Interleukin-12 converts Foxp3+ regulatory $\mathrm{T}$ cells to interferon-gamma-producing Foxp3+ T cells that inhibit colitis. Gastroenterology 140, 2031-2043

69 Schadenberg, A.W. et al. (2011) FOXP3+ CD4+ Tregs lose suppressive potential but remain anergic during transient inflammation in human. European Journal of Immunology 41, 1132-1142

70 Kryczek, I. et al. (2011) IL-17+ regulatory T cells in the microenvironments of chronic inflammation and cancer. Journal of Immunology 186, 4388-4395

71 Dominguez-Villar, M., Baecher-Allan, C.M. and Hafler, D.A. (2011) Identification of Thelper type 1like, Foxp3+ regulatory T cells in human autoimmune disease. Nature Medicine 17, 673-675

72 Pesenacker, A.M. et al. (2013) CD161 defines the subset of FoxP3 + T cells capable of producing proinflammatory cytokines. Blood 121, 2647-2658

73 Beriou, G. et al. (2009) IL-17-producing human peripheral regulatory $\mathrm{T}$ cells retain suppressive function. Blood 113, 4240-4249

74 Voo, K.S. et al. (2009) Identification of IL-17producing FOXP3+ regulatory $\mathrm{T}$ cells in humans. Proceedings of the National Academic Sciences of the United States of America 106, 4793-4798

75 Ayyoub, M. et al. (2009) Human memory FOXP3+ Tregs secrete IL-17 ex vivo and constitutively express the $\mathrm{T}(\mathrm{H}) 17$ lineage-specific transcription factor RORgamma t. Proceedings of the National Academic Sciences of the United States of America $106,8635-8640$
76 Koenecke, C. et al. (2012) IFN-gamma production by allogeneic Foxp3+ regulatory T cells is essential for preventing experimental graft-versus-host disease. Journal of Immunology 189, 2890-2896

77 Sawant, D.V. et al. (2012) Bcl6 controls the Th2 inflammatory activity of regulatory $\mathrm{T}$ cells by repressing Gata3 function. Journal of Immunology 189, 4759-4769

78 Sawitzki, B. et al. (2005) IFN-gamma production by alloantigen-reactive regulatory $\mathrm{T}$ cells is important for their regulatory function in vivo. Journal of Experimental Medicine 201, 1925-1935

79 Wei, C.M. et al. (2008) Frequency and phenotypic analysis of $\mathrm{CD} 4+\mathrm{CD} 25+$ regulatory $\mathrm{T}$ cells in children with juvenile idiopathic arthritis. Journal of Microbiology, Immunology and Infection 41, 78-87

80 Olivito, B. et al. (2009) Th17 transcription factor RORC2 is inversely correlated with FOXP3 expression in the joints of children with juvenile idiopathic arthritis. Journal ofRheumatology 36, 2017-2024

81 de Kleer, I. et al. (2006) Autologous stem cell transplantation for autoimmunity induces immunologic self-tolerance by reprogramming autoreactive T cells and restoring the CD4 + CD25+ immune regulatory network. Blood 107, 1696-1702

82 Macaubas, C. et al. (2010) Distribution of circulating cells in systemic juvenile idiopathic arthritis across disease activity states. Clinical Immunology 134, 206-216

83 Nguyen, K.D. et al. (2011) Serum amyloid A overrides Treg anergy via monocyte-dependent and Treg-intrinsic, SOCS3-associated pathways. Blood 117, 3793-3798

84 Hunter, P.J. et al. (2010) Biologic predictors of extension of oligoarticular juvenile idiopathic arthritis as determined from synovial fluid cellular composition and gene expression. Arthritis \& Rheumatism 62, 896-907

85 Ruprecht, C.R. et al. (2005) Coexpression of CD25 and CD27 identifies FoxP3+ regulatory T cells in inflamed synovia. Journal of Experimental Medicine 201, 1793-1803

86 Raghavan, S. et al. (2009) FOXP3 expression in blood, synovial fluid and synovial tissue during inflammatory arthritis and intra-articular corticosteroid treatment. Annals of the Rheumatic Diseases 68, 1908-1915

87 Bendersky, A. et al. (2012) Cellular interactions of synovial fluid gammadelta $\mathrm{T}$ cells in juvenile idiopathic arthritis. Journal of Immunology 188, 4349-4359 
88 de Jager, W. et al. (2007) Blood and synovial fluid cytokine signatures in patients with juvenile idiopathic arthritis: a cross-sectional study. Annals of the Rheumatic Diseases 66, 589-598

89 Nistala, K. et al. (2010) Th17 plasticity in human autoimmune arthritis is driven by the inflammatory environment. Proceedings of the National Academic Sciences of the United States of America 107, 14751-14756

90 Cosmi, L. et al. (2011) Evidence of the transient nature of the Th17 phenotype of CD4 + CD161+ T cells in the synovial fluid of patients with juvenile idiopathic arthritis. Arthritis \& Rheumatism 63, 2504-2515

91 Thompson, S.D. et al. (2004) A genome-wide scan for juvenile rheumatoid arthritis in affected sibpair families provides evidence of linkage. Arthritis \& Rheumatism 50, 2920-2930

92 Hinks, A. et al. (2010) Association of the AFF3 gene and IL2/IL21 gene region with juvenile idiopathic arthritis. Genes and Immunity 11, 194-198

93 Hinks, A. et al. (2009) Association of the IL2RA/ CD25 gene with juvenile idiopathic arthritis. Arthritis \& Rheumatism 60, 251-257

94 Omoyinmi, E. et al. (2012) Association of the IL-10 gene family locus on chromosome 1 with juvenile idiopathic arthritis (JIA). PLoS One 7, e47673

95 Hinks, A. et al. (2013) Dense genotyping of immune-related disease regions identifies 14 new susceptibility loci for juvenile idiopathic arthritis. Nature Genetics 45, 664-669

96 Eastell, T., Hinks, A. and Thomson, W. (2007) SNPs in the FOXP3 gene region show no association with Juvenile Idiopathic Arthritis in a UK Caucasian population. Rheumatology (Oxford) 46, 1263-1265

97 Prahalad, S. et al. (2008) Lack of association of functional CTLA4 polymorphisms with juvenile idiopathic arthritis. Arthritis \& Rheumatism 58, 2147-2152

98 Frey, O. et al. (2005) The role of regulatory T cells in antigen-induced arthritis: aggravation of arthritis after depletion and amelioration after transfer of CD4 + CD25+ T cells. Arthritis Research \& Therapy 7, R291-R301

99 Killebrew, J.R. et al. (2011) A self-reactive TCR drives the development of Foxp3 + regulatory $\mathrm{T}$ cells that prevent autoimmune disease. Journal of Immunology 187, 861-869

100 Frey, O. et al. (2010) Regulatory T cells control the transition from acute into chronic inflammation in glucose-6-phosphate isomerase-induced arthritis. Annals of Rheumatic Disease 69, 1511-1518

101 Ohkura, N. and Sakaguchi, S. (2010) Regulatory T cells: roles of $\mathrm{T}$ cell receptor for their development and function. Seminars in Immunopathology 32, 95-106

102 Oh, S. et al. (2012) Requirement for diverse TCR specificities determines regulatory $\mathrm{T}$ cell activity in a mouse model of autoimmune arthritis. Journal of Immunology 188, 4171-4180

103 Mavin, E. et al. (2012) Regulatory T cells inhibit CD8+ T-cell tissue invasion in human skin graftversus-host reactions. Transplantation 94, 456-464

104 de Kleer, I.M. et al. (2003) The spontaneous remission of juvenile idiopathic arthritis is characterized by CD30 + T cells directed to human heat-shock protein 60 capable of producing the regulatory cytokine interleukin-10. Arthritis \& Rheumatism 48, 2001-2010

105 Sedlackova, L. et al. (2006) Peripheral blood mononuclear cell responses to heat shock proteins and their derived synthetic peptides in juvenile idiopathic arthritis patients. Inflammation Research 55, 153-159

106 Kamphuis, S. et al. (2005) Tolerogenic immune responses to novel T-cell epitopes from heat-shock protein 60 in juvenile idiopathic arthritis. Lancet $366,50-56$

107 Ojeda, G. et al. (2011) Complement regulatory protein Crry/p65 costimulation expands natural treg cells with enhanced suppressive properties in proteoglycan-induced arthritis. Arthritis \& Rheumatism 63, 1562-1572

108 Gonzalez-Rey, E. et al. (2006) Vasoactive intestinal peptide induces CD4,$+ \mathrm{CD} 25+\mathrm{T}$ regulatory cells with therapeutic effect in collagen-induced arthritis. Arthritis \& Rheumatism 54, 864-876

109 Pozo, D., Anderson, P. and Gonzalez-Rey, E. (2009) Induction of alloantigen-specific human $\mathrm{T}$ regulatory cells by vasoactive intestinal peptide. Journal of Immunology 183, 4346-4359

110 Sun, J. et al. (2012) Superior molecularly altered influenza virus hemagglutinin peptide 308-317 inhibits collagen-induced arthritis by inducing CD4+ Treg cell expansion. Arthritis \& Rheumatism 64, 2158-2168

111 Park, M.K. et al. (2011) Grape seed proanthocyanidin extract (GSPE) differentially regulates Foxp3(+) regulatory and IL-17(+) pathogenic $\mathrm{T}$ cell in autoimmune arthritis. Immunology Letters 135, 50-58

112 Delgado, M. et al. (2001) Vasoactive intestinal peptide prevents experimental arthritis by downregulating both autoimmune and inflammatory components of the disease. Nature Medicine 7, 563-568 
113 Chorny, A. et al. (2005) Vasoactive intestinal peptide induces regulatory dendritic cells with therapeutic effects on autoimmune disorders. Proceedings of the National Academic Sciences of the United States of America 102, 13562-13567

114 Toscano, M.G. et al. (2010) Dendritic cells transduced with lentiviral vectors expressing VIP differentiate into VIP-secreting Tolerogenic-like DCs. Molecular Therapy 18, 1035-1045

115 Fernandez-Martin, A. et al. (2006) Vasoactive intestinal peptide induces regulatory $\mathrm{T}$ cells during experimental autoimmune encephalomyelitis. European Journal of Immunology 36, 318-326

116 Wang, Y. et al. (2007) Vasoactive intestinal polypeptide enhances oral tolerance by regulating both cellular and humoral immune responses. Clinical \& Experimental Immunology 148, 178-187

117 Hamidi, S.A. et al. (2006) Clues to VIP function from knockout mice. Annals of the New York Academy of Sciences 1070, 5-9

118 Chen, G. et al. (2008) The therapeutic effect of vasoactive intestinal peptide on experimental arthritis is associated with CD4 + CD25+ T regulatory cells. Scandinavian Journal of Immunology 68, 572-578

119 Delgado, M. et al. (2008) In vivo delivery of lentiviral vectors expressing vasoactive intestinal peptide complementary DNA as gene therapy for collagen-induced arthritis. Arthritis \& Rheumatism 58, 1026-1037

120 Ganea, D. and Sun, L. (1993) Vasoactive intestinal peptide downregulates the expression of IL-2 but not of IFN gamma from stimulated murine $\mathrm{T}$ lymphocytes. Journal of Neuroimmunology 47, $147-158$

121 Anderson, P. and Gonzalez-Rey, E. (2010) Vasoactive intestinal peptide induces cell cycle arrest and regulatory functions in human T cells at multiple levels. Molecular and Cellular Biology 30, 2537-2551

122 Delgado, M. et al. (2006) Vasoactive intestinal polypeptide induces regulatory dendritic cells that prevent acute graft versus host disease and leukemia relapse after bone marrow transplantation. Annals of the New York Academy of Sciences 1070, 226-232

123 Gonzalez-Rey, E. et al. (2006) Vasoactive intestinal peptide generates human tolerogenic dendritic cells that induce CD4 and CD8 regulatory T cells. Blood 107, 3632-3638

124 Juarranz, Y. et al. (2008) Differential expression of vasoactive intestinal peptide and its functional receptors in human osteoarthritic and rheumatoid synovial fibroblasts. Arthritis \& Rheumatism 58, 1086-1095

125 Yukawa, T. et al. (2007) Differential expression of vasoactive intestinal peptide receptor 1 expression in inflammatory bowel disease. International Journal of Molecular Medicine 20, 161-167

126 Delgado, M. et al. (2008) Genetic association of vasoactive intestinal peptide receptor with rheumatoid arthritis: altered expression and signal in immune cells. Arthritis \& Rheumatism 58, 1010-1019

127 Pesenacker, A.M. et al. (2010) The role of vasoactive intestinal peptide receptor type one in pathogenesis of oligoarticular juvenile idiopathic arthritis. Presented at the British Society of Immunology Congress (2010; Liverpool)

128 Onoue, S. et al. (2012) Inhalable sustained-release formulation of long-acting vasoactive intestinal peptide derivative alleviates acute airway inflammation. Peptides 35, 182-189

129 Harmar, A.J. et al. (2012) Pharmacology and functions of receptors for vasoactive intestinal peptide and pituitary adenylate cyclase-activating polypeptide: IUPHAR review 1. British Journal of Pharmacology 166, 4-17

130 Wing, K. et al. (2008) CTLA-4 control over Foxp3+ regulatory T cell function. Science 322, 271-275

131 Flores-Borja, F. et al. (2008) Defects in CTLA-4 are associated with abnormal regulatory T cell function in rheumatoid arthritis. Proceedings of the National Academy of Sciences of the United States of America 105, 19396-19401

132 Ko, H.J. et al. (2010) CTLA4-Ig modifies dendritic cells from mice with collagen-induced arthritis to increase the CD4 + CD25 + Foxp3+ regulatory T cell population. Journal of Autoimmunity 34, $111-120$

133 Ruderman, E.M. and Pope, R.M. (2005) The evolving clinical profile of abatacept (CTLA4-Ig): a novel co-stimulatory modulator for the treatment of rheumatoid arthritis. Arthritis Research \& Therapy 7(Suppl 2), S21-S25

134 Schiff, M. et al. (2008) Efficacy and safety of abatacept or infliximab vs placebo in ATTEST: a phase III, multi-centre, randomised, double-blind, placebo-controlled study in patients with rheumatoid arthritis and an inadequate response to methotrexate. Annals of the Rheumatic Disease 67, 1096-1103

135 Ruperto, N. et al. (2008) Abatacept in children with juvenile idiopathic arthritis: a randomised, double-

Accession information: doi:10.1017/erm.2013.14; Vol. 15; e13; November 2013 (C) Cambridge University Press 2013. The online version of this article is published within an Open Access environment subject to the conditions of the Creative Commons Attribution licence http://creativecommons.org/licenses/by/3.0/. 
blind, placebo-controlled withdrawal trial. Lancet 372, 383-391

136 Alvarez-Quiroga, C. et al. (2011) CTLA-4-Ig therapy diminishes the frequency but enhances the function of Treg cells in patients with rheumatoid arthritis. Journal of Clinical Immunology 31, 588-595

137 Bluestone, J.A. et al. (2008) The effect of costimulatory and interleukin 2 receptor blockade on regulatory $\mathrm{T}$ cells in renal transplantation. American Journal of Transplantation 8, 2086-2096

138 Charbonnier, L.M. et al. (2012) CTLA4-Ig restores rejection of MHC class-II mismatched allografts by disabling IL-2-expanded regulatory $\mathrm{T}$ cells. American Journal of Transplantation 12, 2313-2321

139 Riella, L.V. et al. (2012) Deleterious effect of CTLA4Ig on a Treg-dependent transplant model. American Journal of Transplantation 12, 846-855

140 St Clair, E.W. (2008) The calm after the cytokine storm: lessons from the TGN1412 trial. Journal of Clinical Investigation 118, 1344-1347

141 Biton, J. et al. (2011) Interplay between TNF and regulatory $\mathrm{T}$ cells in a TNF-driven murine model of arthritis. Journal of Immunology 186, 3899-3910

142 Ehrenstein, M.R. et al. (2004) Compromised function of regulatory T cells in rheumatoid arthritis and reversal by anti-TNFalpha therapy. Journal of Experimental Medicine 200, 277-285

143 Valencia, X. et al. (2006) TNF downmodulates the function of human CD4 + CD25hi T-regulatory cells. Blood 108, 253-261

144 Wang, J. et al. (2008) Suppressor activity among CD4 + , CD25 + + T cells is discriminated by membrane-bound tumor necrosis factor alpha. Arthritis \& Rheumatism 58, 1609-1618

145 Huang, B. et al. (2012) Combined use of etanercept and MTX restores CD4(+)/CD8 (+) ratio and Tregs in spleen and thymus in collagen-induced arthritis. Inflammation Research 61, 1229-1239

146 van der Heijde, D. et al. (2006) Comparison of etanercept and methotrexate, alone and combined, in the treatment of rheumatoid arthritis: two-year clinical and radiographic results from the TEMPO study, a double-blind, randomized trial. Arthritis \& Rheumatism 54, 1063-1074

147 Antoni, C. and Kalden, J.R. (1999) Combination therapy of the chimeric monoclonal anti-tumor necrosis factor alpha antibody (infliximab) with methotrexate in patients with rheumatoid arthritis. Clinical \& Experimental Rheumatology 17(Suppl 18), S73-S77
148 Huang, Z. et al. (2012) Anti-TNF-alpha therapy improves Treg and suppresses Teff in patients with rheumatoid arthritis. Cellular Immunology 279, 25-29

149 Lina, C. et al. (2011) Combined treatment of Etanercept and MTX reverses Th1/Th2, Th17/ Treg imbalance in patients with rheumatoid arthritis. Journal of Clinical Immunology 31, 596-605

150 Blache, C. et al. (2011) Number and phenotype of rheumatoid arthritis patients' CD4 + CD25hi regulatory T cells are not affected by adalimumab or etanercept. Rheumatology (Oxford) 50, 1814-1822

151 Dige, A. et al. (2011) Adalimumab treatment in Crohn's disease does not induce early changes in regulatory T cells. Scandinavian Journal of Gastroenterology 46, 1206-1214

152 McGovern, J.L. et al. (2012) Th17 cells are restrained by Treg cells via the inhibition of interleukin- 6 in patients with rheumatoid arthritis responding to anti-tumor necrosis factor antibody therapy.

Arthritis \& Rheumatism 64, 3129-3138

153 Feng, G., Wood, K.J. and Bushell, A. (2008) Interferon-gamma conditioning ex vivo generates CD25 + CD62L + Foxp3+ regulatory T cells that prevent allograft rejection: potential avenues for cellular therapy. Transplantation 86, 578-589

154 Duarte, J.H. et al. (2009) Natural Treg cells spontaneously differentiate into pathogenic helper cells in lymphopenic conditions. European Journal of Immunology 39, 948-955

155 De Kleer, I.M. et al. (2004) Autologous stem cell transplantation for refractory juvenile idiopathic arthritis: analysis of clinical effects, mortality, and transplant related morbidity. Annals of the Rheumatic Diseases 63, 1318-1326

156 Wulffraat, N.M. et al. (2003) Long-term follow-up of autologous stem cell transplantation for refractory juvenile idiopathic arthritis. Bone Marrow Transplantation 32(Suppl 1)S61-S64

157 Abinun, M. et al. (2009) Autologous T cell depleted haematopoietic stem cell transplantation in children with severe juvenile idiopathic arthritis in the UK (2000-2007). Molecular Immunology 47, 46-51

$158 \mathrm{Wu}, \mathrm{Q}$. et al. (2013) T cell depleted autologous stem cell transplantation results in a chimera of clones from before and after transplant in systemic juvenile idiopathic arthritis. Presented at the Rheumatology (2013; Birmingham)

159 Swart, J., Martens, A. and Wulffraat, N. (2008) Mesenchymal stem cells: a future for the treatment of arthritis? Joint Bone Spine 75, 379-382 
160 Calkoen, F.G. et al. (2013) Mesenchymal stromal cells isolated from children with systemic juvenile idiopathic arthritis suppress innate and adaptive immune responses. Cytotherapy 15, 280-291
161 Gonzalez, M.A. et al. (2009) Treatment of experimental arthritis by inducing immune tolerance with human adipose-derived mesenchymal stem cells. Arthritis \& Rheumatism 60, 1006-1019

\section{Further reading, resources and contacts Clinical trail databases}

Clinical trials registry of the U.S. National Institutes of Health clinicaltrials.gov

EU Clinical Trials Register: $\quad$ www.clinicaltrialsregister.eu/ctr-search/search

\section{Features associated with this article}

\section{Figures}

Figure 1. Regulatory T cell families.

Figure 2. Proposed Treg-Tconv balance in juvenile idiopathic arthritis (JIA).

Table

Table 1. Evidence for the role or dysfunction of Treg in JIA.

\section{Citation details for this article}

Anne M. Pesenacker and Lucy R. Wedderburn (2013) T regulatory cells in childhood arthritis - novel insights. Expert Rev. Mol. Med. Vol. 15, e13, November 2013, doi:10.1017/erm.2013.14 